\title{
Análise dos perfis e das seções resistivas de poço na caracterização de um reservatório carbonático da Bacia de Santos
}

\author{
Herson Rocha1'2, Abel Carrasquilla², Jéssica Lia Costa ${ }^{2}$, Alfredo Carrasco ${ }^{3},{ }^{1}$ UFRA, Parauapebas - PA, Brasil,
} 2LENEP/UENF, Macaé - RJ, Brasil, 3UFF, Niterói - RJ, Brasil.

Copyright 2018, SBGf - Sociedade Brasileira de Geofísica

Este texto foi preparado para a apresentação no VIII Simpósio Brasileiro de Geofísica, Salinópolis, 18 a 20 de setembro de 2018. Seu conteúdo foi revisado pelo Comitê Técnico do VIII SimBGf, mas não necessariamente representa a opinião da SBGf ou de seus associados. É proibida a reprodução total ou parcial deste material para propósitos comerciais sem prévia autorização da SBGf.

\section{Resumo}

This study aims to analyze petrophysical properties and construct resistivity sections from the interpretation of the geophysical profiles of a pre-salt carbonate reservoir in the Santos, southeast Basin of Brazil. In the interpretation, it was possible to characterize the reservoir in 6 flow units and to elaborate two sections of resistivity, which were based on the logs of the lateral profiles and induction, which examine the resistivity variation inside the reservoir in surface resistivity (LLS), medium LLM) and deep (LLD). However, both responses of the profiles presented higher values of resistivity in the zones closer to the well wall, which occurs due to the invasion of the drilling mud, since these layers present values of porosity and permeability in laboratory tests ranging from 2 to $20 \%$ and 0.1 to $900 \mathrm{mD}$, respectively. The methodology adopted was effective to identify the heterogeneities and the permo-porous zones in this reservoir

\section{Introdução}

O objetivo principal deste trabalho foi analisar as propriedades petrofísicas e construir seções de resistividade em um reservatório carbonático do pré-sal brasileiro, tentando identificar horizontes promissores para exploração e produção de petróleo. As medidas de porosidade e permeabilidade, obtidas de amostras de laboratório, foram confrontadas com perfis de poços para verificar suas coincidências. As seções de resistividade foram baseadas em registros dos perfis laterolog e indução, que fornecem distâncias rasas, médias e profundas de investigação dentro do reservatório.

O petróleo é encontrado no interior do espaço poroso das rochas reservatórios, ou seja, rocha que apresenta porosidade e permeabilidade, permitindo 0 armazenamento e fluxo do fluido no interior da rocha (Tucker M, 2009). Segundo Carvalho et al. (2014), a porosidade e permeabilidade são de relevante importância na caracterização de um reservatório e determinação de padrões de fluxo, com o objetivo de otimizar a produção de petróleo.

As complexidades do sistema poroso apresentadas nos reservatórios carbonáticos, especialmente as do pré-sal brasileiro, faz com que suas propriedades permo-porosas sejam difíceis de quantificar devido à variação na escala de meios porosos. No entanto, utilizando imagens de perfis de resistividade para verificar a penetração do fluido de perfuração, é possível analisar heterogeneidades em escala e obter dados qualitativos e representativos.

Assim, perfis de poços e análises de amostras de rochas em laboratório são métodos amplamente utilizados para avaliar as propriedades físicas de formações geológicas na caracterização petrofísica de reservatórios carbonáticos (Aguilera \& Aguilera, 2001). A resolução de dados e a cobertura espacial nestes dois métodos, combinados com a quantidade de parâmetros medidos, ocorrem em diferentes faixas para obter conhecimento da informação estrutural litológica e subsuperficial (Shenawi et al., 2007).

Os estudos foram estabelecidos na Formação Barra Velha, na Bacia de Santos, que é subdividida em sequências superior e inferior. A inferior data do início do aptiano e é caracterizada pela deposição de calcários microbianos, estromatólitos e laminitos nas seções proximais e xistos nas porções mais distantes. Há também grainstones e packstones compostos de fragmentos de estromatólito e bioclasto. A seqüência superior data do Aptiano tardio, onde existem calcários estromatolíticos e laminitos microbianos dolomitizados (Moreira et al., 2007).

\section{Metodologia/ Problema Investigado}

O conjunto de dados fornecidos para o desenvolvimento deste estudo partiu de um trecho de $25 \mathrm{~m}$ do reservatório carbonático - representados pelas profundidades 575 até 600 (valores fictícios) para preservar a identidade do poço. Nesses dados, continham informações geológicas, medições laboratoriais, conjuntos básicos de perfis de poços (raio gama - GR, porosidade de nêutrons - NPHI, densidade - $\mathrm{RHOB}$, laterolog e registros de resistividade de indução), e perfis especiais, tais como o perfil de ressonância magnética nuclear - NMR.

Com essas informações, a primeira iniciativa foi caracterizar o reservatório em unidades de fluxo (UF) proposto por Tiab \& Donaldson (2012). Em seguida utilizou-se a metodologia de Winland (1972) com a finalidade de identificar o tamanho do raio das gargantas de poro. Após estas etapas, foram analisados os perfis laterolog de resistividade superficial, média e profunda (LLS, LLM e LLD, respectivamente) e seus valores foram interpolados, por meio de um polinômio cúbico, para construir uma seção de resistividade. De modo similar, a mesma abordagem foi utilizada para os perfis de resistividade por indução - Array Induction Tool - AIT (10, 20, 30, 60 e 90), no qual também foi construída uma seção de resistividade para imagear, de maneira radial o 
poço e analisar as zonas mais invadidas pela lama de perfuração. Por outro lado, os dados de NMR foram utilizados para estimar a porosidade e a permeabilidade, que foram comparadas com as medidas laboratoriais. Finalmente, uma interpretação integrada abrangendo todas as interpretações individuais foi realizada. Todas as análises, interpretações e manuseio dos dados foram feitos utilizando os softwares Interactive Petrophysics (IP, 2016), Excel (EXCEL, 2016) e MATLAB (MATLAB, 2016).

\section{Resultados}

Primeiramente aplicamos a correlação entre a porosidade em função da permeabilidade a partir dos valores extraídos das amostras de plug (Figura 1, parte superior) Em seguida costrui-se o Gráfico de Lorenz Modificado para identificar as zonas de fluxo com a finalidade de caracterizar o resservatório. Nesta abordagem, notou-se a presença de 6 zonas (Z1, Z2, Z3, Z4, Z5 e Z6), sendo que as cinco primeiras são zonas onde a capaciade de deslocamento de fluxo é maior que a capacidade de armazenamento e na Z6 ocorre o contrario, ou seja, a capacidade de armazenamento é maior que a capacidade de fluxo, configurando assim uma zona de barreira (Figura 1, parte inferior).

Os perfis laterog apresentaram valores de resistividade variando de 0,2 a 1995 ohm.m entre 583 e 593 m de profundidade, com uma zona com altos valores de resistividade nos registros LLS e LLM e menores valores de RT nas curvas LLD e na resistividade da formação (RT) (Figura 2a). Segundo Crain (1984), esse cenário caracteriza uma invasão de lama no reservatório, quando a formação geológica apresenta hidrocarbonetos e fluidos da água de formação. No caso dos perfis de indução (AIT), a mesma zona de alta resistividade identificada pelos logs LLS e LLM também foi identificada pelos perfis AO10, AO20 e AO30 (Figura 2b). Os registros $A 060$ e AO90 mostraram valores de $\mathrm{Rt}$ mais altos que o LLD e um formato similar. Observa-se que o pico superior aparece em todas as curvas do laterolog, na curva Rt e em todas os perfis de indução. O pico inferior aparece apenas nos perfis LLD e A090, assim como na curva Rt, ou seja, é uma anomalia profunda no reservatório. Ambos os picos podem representar informação litológica associadas a zonas de fraturadas no reservatório.

A partir disso, as abordagens de Amaefule et al. (1993) e Winland (1972) foram usadas para dividir o reservatório em 6 unidades de fluxo (track 2 na Figura 3). Esta abordagem permitiu identificar a paRte superior e inferior do reservatório, o contato oléo - água e as zonas permoporosas. O perfil de raios gama (track 3 na Figura 3) mostra baixa concentração de argila entre 582 e 594 m de profundidade, assim como o track 5 exibe a litologia, considerando principalmente o volume de argila $\left(\mathrm{V}_{\mathrm{sh}}\right)$, maior concentração de carbonatos e menor saturação de água $\left(S_{w}\right)$ nos valores de $S_{w}$ superiores e superiores, acima e abaixo, que correspondem ao intervalo do reservatório. No mesmo intervalo, os perfis de resistividade, laterolog e indução (track 6 e 8), agrupados com a resistividade da formação $\left(R_{t}\right)$, bem como as seções de resistividade, mostram valores altos na mesma zona do reservatório, o que permite inferir a a presença de óleo (track 7 e 9). É possível identificar, com mais detalhes, as áreas mais invadidas pela lama e as heterogeneidades do reservatório.

O track 10 mostra um cruzamento entre os perfis RHOB e NPHI, o que caracteriza a presença de hidrocarbonetos nestas profundidades. Por outro lado, no track 11 observa-se as medições laboratoriais de porosidade e permeabilidade deste reservatório, evidenciando valores mais elevados na zona de óleo em relação à zona aqüífera, talvez devido à maior presença de reações químicas na água que causam dissolução e cimentação. A partir do corte de distribuição T2 do perfil de NMR (track 14), o reservatório foi dividido em 5 zonas (track 12), descartando-se o topo (575 a $581 \mathrm{~m})$ devido à falta de informações para comparação. Observa-se as coincidências dessa divisão do reservatório em relação às zonas de fluxo no track 2. Já o track 13 mostra as medições laboratoriais de permeabilidade $e$ as estimativas desse parâmetro usando o perfil NMR com as abordagens SDR de Kenyon (1997) e a de Timur (1968). As avaliações de permeabilidade mostraram uma tendencia próxima em relação aos dados laboratóriais, no entanto, na faixa entre 589 - 594 m, não verificou-se a mesma a tendência, sendo necessário análisar outros valores deste parâmetro dentro do reservatório.

\section{Discussão e Conclusões}

Do ponto de vista petrofísico, a abordagem assumida neste estudo permitiu uma boa caracterização do reservatório em unidades de fluxo hidráulico. Com isso, as medidas de resistividade mostraram claramente a invasão da lama de perfuração dentro do reservatório através dos altos valores de resistividade nas menores profundidades de investigação observados principalmente nos perfis laterolog.

A partir da análise conjunta dos dados de resistividade laterolog e de indução - foi possível construir seções de resistividade para inferir o quanto o meio geológico é heterogêneo, o que também pode ser identificado em outros registros e nos testes de amostra de laboratório.

As seções provaram ser uma ferramenta útil na interpretação do reservatório, especialmente na interpretação conjunta com outros perfis, embora, seja importante reconhecer as limitações da ferramenta para medir valores muito altos de resistividade.

A caracterização do reservatório em unidades de fluxo praticamente coincidiu com o zoneamento realizado por NMR, o que valida a metodologia proposta, possibilitando analisar os intervalos de interesse econômico dentro do reservatório.

\section{Agradecimentos}

À UENF pela estrutura e supoRte computacional, CAPES e PRH-226 Petrobras / UENF para bolsas concedidas, Galp Petrogal Brasil pelo conjunto de dados fornecidos, e o primeiro autor também estende seus agradecimentos à UFRA pela licença concedida para qualificação profissional. 


\section{Referências}

Aguilera, R. \& Aguilera, M. 2001. The integration of capillary pressures and Pickett plots for determination. SPE Conference, paper no. 71725.

Amaefule, J.; Altunbay, M.; Tiab, D., Kersey, D. \& Keelan, D. 1993. Enhanced reservoir description using core and log data to identify hydraulic (flow) units and predict permeability in uncored intervals/wells. SOC. Petrol. Eng. Paper No. 26436.

Carvalho, S., Oliveira, M., Duarte, T., Duarte, M., \& Barros Neto, E. (2014). Blucher Chemical Engineering Proceedings, v. 1, n. 2, p. 13739-13745.

Crain, E. 1984. Log analysis handbook Vol. 1: Quantitative Log Analysis. Pennwell Books, Tulsa, OK. (https://www.spec2000.net/05-bibliography.htm consulted on January 5,2018$)$.

MICROSOFT EXCEL. 2016. Users' Manual.

IP. 2015. Interactive Petrophysics Users' Manual.

Kenyon, W. 1997. Petrophysical principles of applications of NMR logging. The Log Analyst 38 (2): 21-43.

MATLAB. 2016. MATLAB \& Simulink User's Manual.

Moreira, J.; Madeira, C.; Gil, J. \& Machado, M. 2007. Santos Basin. Petrobras Geosciences Bulletin, Rio de Janeiro, v. 15, n. 2, p. 531-549. (In Portuguese).

Shenawi, S.; White, J.; Elrafie, E. \& El-Kilany, K. 2007. Permeability and water saturation distribution by lithologic facies and hydraulic units: a reservoir simulation case study. Presented at the SPE Middle East Oil and Gas Show and Conference, Manama, Bahrain, SPE105273.

Tiab, D. \& Donaldson, E. 2012. Petrophysics: Theory and practice of measuring reservoir rock and fluid transport properties. Gulf Professional Publishing, 3rd Edition, 950 p.

Timur, A. 1968. An Investigation of Permeability, Porosity, and Residual Water Saturation Relationships for Sandstone Reservoirs. The Log Analyst 9 (4): 8.

Tucker, M. (2009). Sedimentary petrology: an introduction to the origin of sedimentary rocks. John Wiley \& Sons.

Winland, H. 1972. Oil accumulation in response to pore size charges, Weyburn field, Saskatchewan. Amoco Production Company Research Department, F72-G-25. 


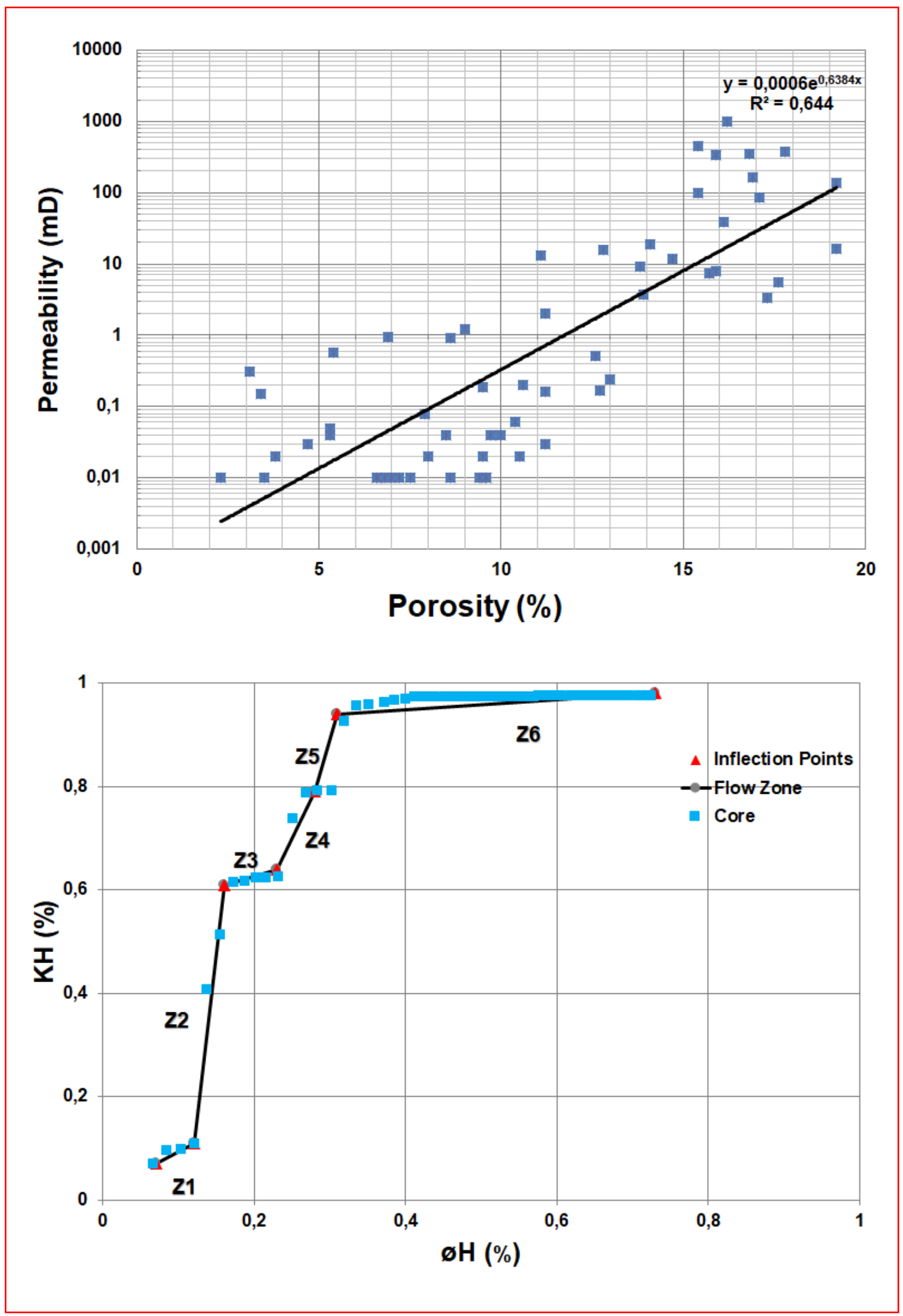

Figura 1 - Correlação entre porosidade e permeabilidade (parte superior) e Gráfico de Lorenz Modificado baseado nos pontos de inflexão das zonas de fluxo (parte inferior). 


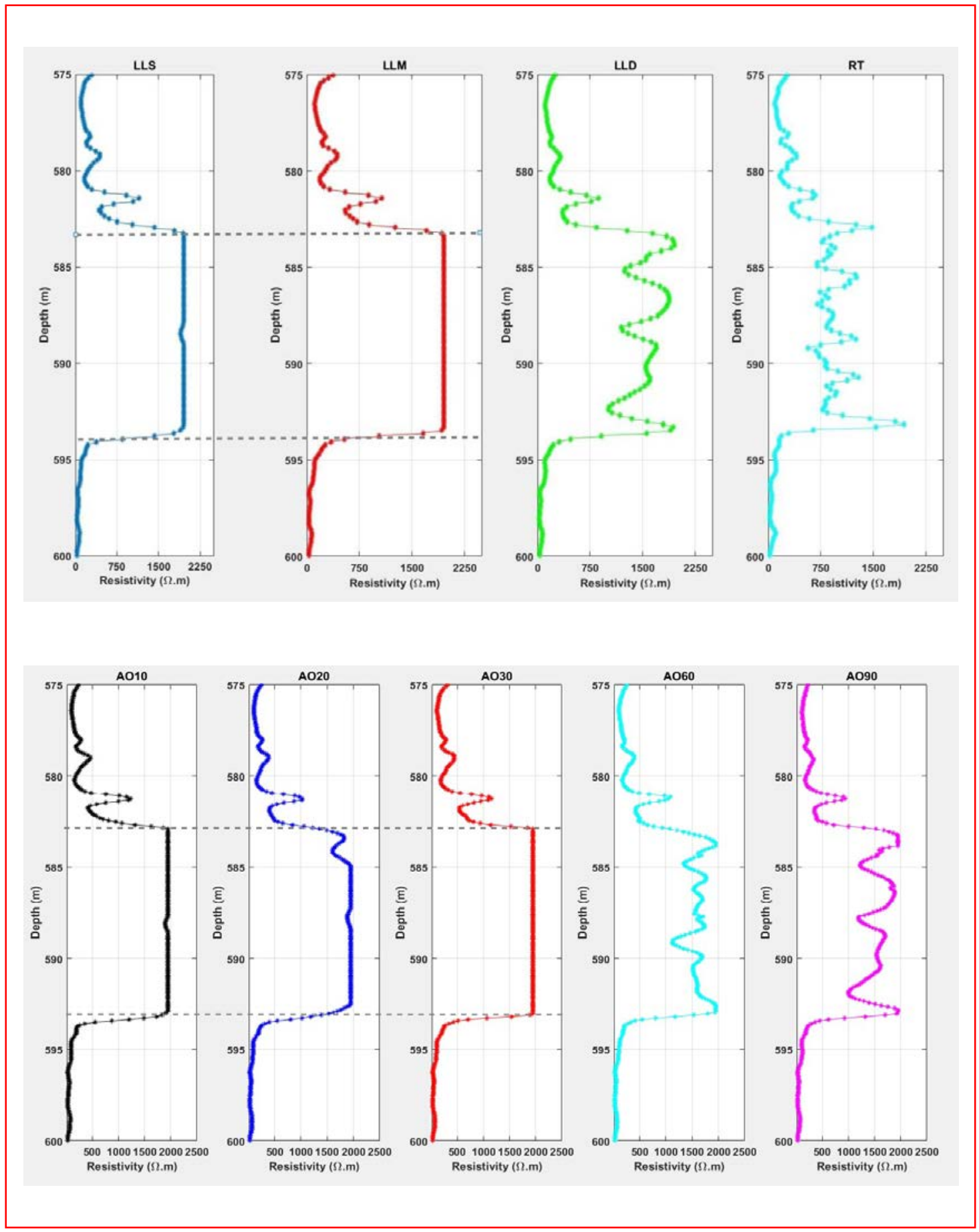

Figura 2 - Perfis de resistividade laterolog LLS, LLM e LLD nos tracks 1 a 3 e a resistividade da formação (RT) no track 4 (parte superior). Perfis de resistividade AIT com arranjos de 10, 20, 30, 60 e 90 nos tracks 1 a 5, respectivamente (parte inferior). 


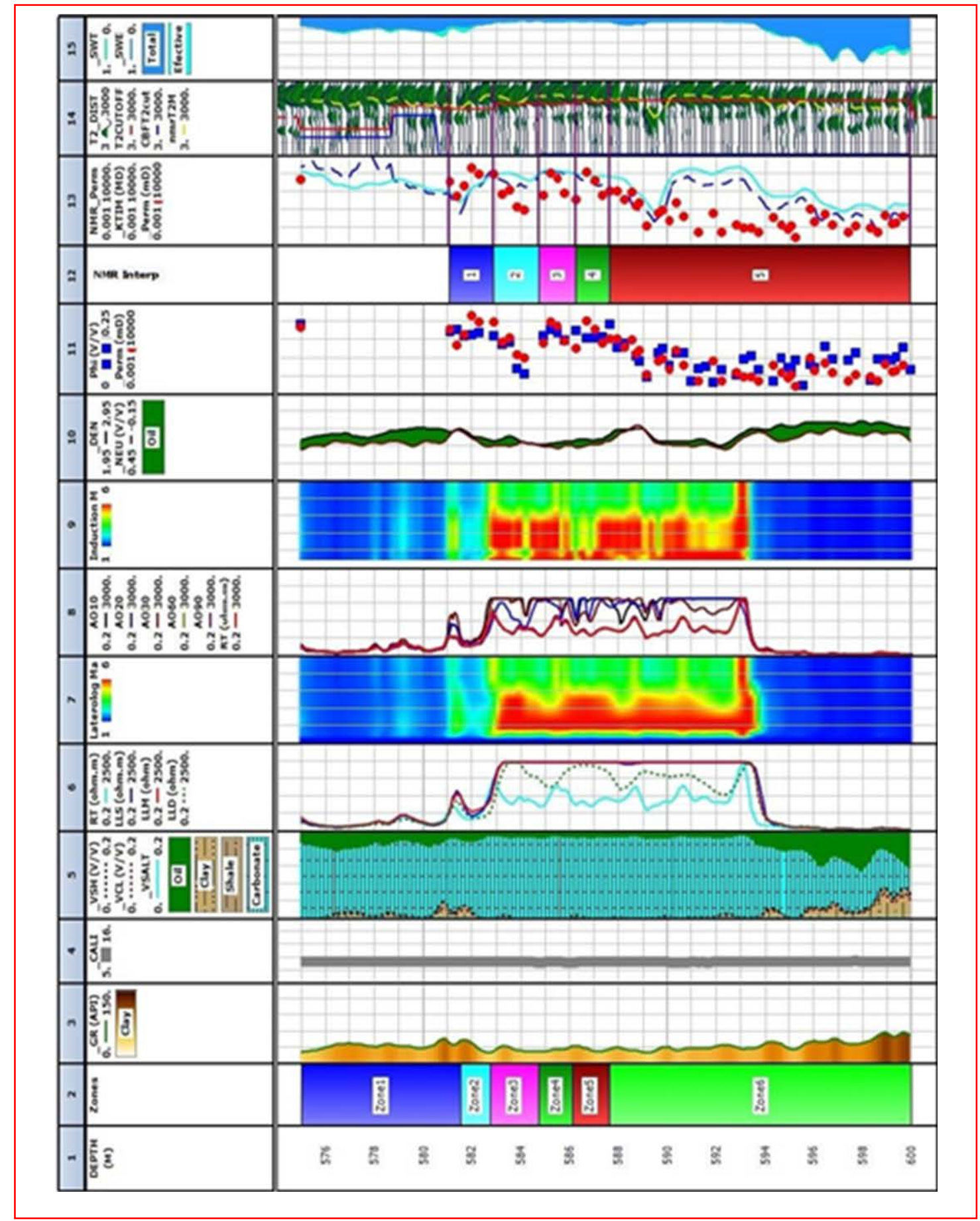

Figura 3 - Profundidade (track 1); perfis básicos (gamma ray no track 3, caliper no track 4), perfis de resistividade laterolog e indução (track 6 e 8), densidade e porosidade neutrônica (track 10), junto coma as zonas de fluxo (track 2); litologia (track 5) e saturação de água (track 15);seção de resistividade dos perfis laterolog (track 7); seção de resistividade dos perfis de indução (track 9); porosidade e permeabilidade laboratorial (track 11); divisão do reservatório por NMR (track 12); permeabilidade laboratorial medida e estimada (track 13) e distribuição NMR dos tempo de corte $T_{2}$ (track 14). 